\title{
Changes in optical coherence tomography measurements after orbital wall decompression in dysthyroid optic neuropathy
}

\author{
Kyung-Ah Park ${ }^{1} \cdot$ Yoon-Duck Kim ${ }^{1} \cdot$ Kyung In Woo ${ }^{1}$
}

Received: 14 February 2017 / Revised: 25 October 2017 / Accepted: 21 December 2017 / Published online: 1 March 2018

(c) The Royal College of Ophthalmologists 2018

\begin{abstract}
Purpose The purpose of our study was to assess changes in peripapillary retinal nerve fiber layer (RNFL) thickness after orbital wall decompression in eyes with dysthyroid optic neuropathy (DON).

Methods We analyzed peripapillary optical coherence tomography (OCT) images (Cirrus HD-OCT) from controls and patients with DON before and 1 and 6 months after orbital wall decompression.

Results There was no significant difference in mean preoperative peripapillary retinal nerve fiber layer thickness between eyes with DON and controls. The superior and inferior peripapillary RNFL thickness decreased significantly 1 month after decompression surgery compared to preoperative values $(p=0.043$ and $p=0.022$, respectively). The global average, superior, temporal, and inferior peripapillary RNFL thickness decreased significantly 6 months after decompression surgery compared to preoperative values $(p=0.015, p=0.028, p=0.009$, and $p=0.006$, respectively). Patients with greater preoperative inferior peripapillary RNFL thickness tended to have better postoperative visual acuity at the last visit $(p=$ 0.024 , OR $=0.926$ ).

Conclusions Our data revealed a significant decrease in peripapillary RNFL thickness postoperatively after orbital decompression surgery in patients with DON. We also found that greater preoperative inferior peripapillary RNFL thickness was associated with better visual outcomes. We suggest that RNFL thickness can be used as a prognostic factor for DON before decompression surgery.
\end{abstract}

\section{Introduction}

Orbital decompression is a well-established treatment for dysthyroid optic neuropathy (DON) [1]. Steroids and radiotherapy may also be used in the acute stages of thyroid orbitopathy, whereas orbital decompression is generally reserved for unremitting optic neuropathy $[2,3]$. The fundamental basis of the surgical approach to DON is that DON can be the result of crowding around the orbital apex with optic nerve compression secondary to an increased volume of orbital soft tissue, such as extraocular muscles [4]. Also, other mechanisms, including compression of the blood supply to the optic nerve [5], an increase in retrobulbar pressure [6], or, rarely, optic nerve stretch [7], have

Yoon-Duck Kim

ydkimoph@skku.edu

1 Department of Ophthalmology, Samsung Medical Center, Sungkyunkwan University School of Medicine, Seoul, Korea been proposed as pathogenic mechanisms of optic nerve damage.

Surgical decompression of the orbit has been performed successfully for nearly a century. Orbital decompression leads to a decrease in proptosis and improvements in visual acuity and optic nerve function in most studies [3, 8-11]. Disc swelling may accompany DON to varying degrees; the reported prevalence of disc swelling is $30-33 \%$ [12, 13]. Garrity et al. [14, 15] previously reported that disc swelling completely resolved in $67 \%$ and improved in $27 \%$ of dysthyroid orbitopathy patients with disc swelling after orbital decompression. Optic disc pallor may also accompany DON in $4-20 \%$ of cases.

The authors previously found that temporal peripapillary retinal nerve fiber layer (RNFL) thickness was less in patients with chronic DON compared to controls and patients with acute DON using optical coherence tomography (OCT) [16]. There was a significant association between RNFL thickness and visual acuity after treatment for dysthyroid orbitopathy. However, in this study, heterogeneous treatment methods were applied and many patients 
received treatment for DON before OCT examination. As a result, the authors could not evaluate the effect of each treatment modality on the OCT measurements. The purpose of the current study was to assess changes in peripapillary RNFL thickness after orbital decompression in eyes with dysthyroid optic neuropathy (DON) and to analyze whether preoperative OCT findings are predictive factors for postoperative visual outcomes.

\section{Methods}

This retrospective study was performed at a single center according to the tenets of the Declaration of Helsinki. This study was approved by the Institutional Review Board of Samsung Medical Center (Seoul, Republic of Korea). We retrospectively reviewed the medical records and OCT measurements of all consecutive cases of DON who underwent orbital decompression surgery by a single surgeon (Y.-D.K.) at the Ophthalmology Department between February 2011 and April 2016. A diagnosis of DON was made clinically based on decreased visual acuity, loss of color vision, relative afferent pupillary defect if one eye was involved, compatible visual field defects, and significant crowding of the orbital apex evident on computed tomography scans. All subjects were previously examined by an endocrinologist and had laboratory evidence of thyroid dysfunction. Exclusion criteria were being 18 years or less in age, previous eye trauma or eye surgery not related to dysthyroid orbitopathy except for uneventful simple cataract extraction, and other ocular pathologies that may affect vision or OCT measurements, including the presence of amblyopia, corneal ulcer, significant media opacities, glaucoma, and retinal disease. Additionally, patients with refractive error greater than 6.0 diopters of spherical equivalent or 3.0 diopters of astigmatism in either eye were excluded because of possible differences in OCT parameters regardless of optic atrophy $[17,18]$. Some patients participated in a cross-sectional study of peripapillary RNFL thickness in patients with DON in the same department [16].

Patient demographics and clinical data were collected. Clinical outcome measures included best-corrected visual acuity, mean deviation on a Humphrey Field Analyzer, color vision test score using Ishihara plates, Hertel exophthalmometry measurements, and peripapillary RNFL thicknesses measured by OCT. Best-corrected visual acuities were measured using trial lenses based on the results of manifest refraction performed using retinoscopy. Then, the visual acuities were transformed to a logarithmic scale ( $\log$ MAR) for statistical analysis. Color vision was tested using Ishihara charts. Color vision score was recorded as the number of correctly identified tables. The visual field was tested using a Humphrey Field Analyzer using the 30-2 SITA-standard protocol (Humphrey 740 Visual Field Analyzer, Carl Zeiss Meditec Inc.). Only reliable visual fields were considered $(\leq 33 \%$ false positives or false negatives; fixation losses $<20 \%$ ), and mean deviation was recorded. Demographics and clinical data of healthy subjects were also collected. All patients and healthy control subjects underwent peripapillary OCT scanning with a Cirrus HDOCT (Carl Zeiss Meditec AG, Jena, Germany) at the initial visit and at 1 and 6 months after orbital wall decompression. Peripapillary RNFL thickness was obtained using the optic disc cube $200 \times 200$ protocol in the Cirrus software (software version 3.0.0.64). This protocol generates a cube of data through a $6 \mathrm{~mm}$ square grid. A $3.46 \mathrm{~mm}$ diameter circle is automatically centered on the optic disc. This analysis provides average RNFL thickness and maps with four quadrants (superior, inferior, nasal, and temporal) and 12 clock-hours, and allows for classification compared to an internal normative database. The operator checked that the OCT software had centered the measurement circle correctly and that it had correctly identified the RNFL limits. To be included, all scans had to have a signal strength $\geq 6$ and no movement artifacts.

The primary indication for orbital decompression was a poor response to intravenous methylprednisolone pulse therapy $(250 \mathrm{mg}$ every $6 \mathrm{~h}$ via intravenous infusion for 3 consecutive days). Patients in this study underwent medial wall orbital decompression via transcaruncular or transnasal endoscopic approaches. After decompression surgery, 80 $\mathrm{mg}$ of prednisone was orally administered for 7 days and tapered at $10 \mathrm{mg}$ per week until a dose of $20 \mathrm{mg}$ was reached, then tapered by $5 \mathrm{mg}$ per week. Orbital radiation therapy was used in patients whose optic neuropathy recurred or when orbital inflammation persisted despite orbital decompression. The orbital radiation dose was 20 Gray in 10 fractions over 2 weeks.

Patients were asked to attend follow-up visits 1, 3, and 6 months postoperatively and then every year thereafter. At every visit, best-corrected visual acuity, visual field, color vision, exophthalmos, and peripapillary RNFL thicknesses were evaluated.

Statistical analyses were performed using the Statistical Analysis System version 9.4 (SAS Institute Inc., Cary, NC, USA). Age was compared between patients with DON and control using Student's $t$-test. Gender was compared between patients with DON and control using Fisher's exact test. Baseline peripapillary RNFL thickness ate the last preoperative visit was compared between patients with DON and control using a Generalized Estimating Equations approach [19]. The pre- and post-operation thickness of peripapillary RNFL was compared also using a Generalized Estimating Equations approach. Baseline factors associated with post-operative visual acuity at the last visit were 
Table 1 Demographics and initial ophthalmic examinations in controls and patients with dysthyroid optic neuropathy

\begin{tabular}{lcc}
\hline Variable & $\begin{array}{c}\text { Controls }(n=47,94 \\
\text { eyes }) \\
\text { Mean } \pm \text { SD }\end{array}$ & $\begin{array}{c}\text { DON }(n=11,20 \\
\text { eyes }) \\
\text { Mean } \pm \text { SD }\end{array}$ \\
\hline Age (years) & $48 \pm 14$ & $54 \pm 12$ \\
Gender (male/female) & $26 / 21$ & $2 / 9$ \\
Smokers ( $n$ ) & & $3(27 \%)$ \\
Follow-up duration (months) & $23 \pm 17$ \\
Initial LogMAR visual acuity & & $0.043^{\dagger}$ \\
Color vision test (Ishihara) score & & $5 \pm \pm 0.46$ \\
Hertel exophthalmometry (mm) & $18 \pm 4$ \\
Mean deviation on Humphrey field analyzer & $-7.8 \pm 4.5$ \\
(dB) & & 5.5
\end{tabular}

*Student's $t$-test

†Fisher's exact test

${ }^{\ddagger}$ Humphrey Field Analyzer using the 30-2 SITA-standard protocol

analyzed using a univariate and multivariable analyses. A $p$ value $<0.05$ was taken to indicate statistical significance.

\section{Results}

Sixteen consecutive patients met the inclusion criteria. Eleven patients (20 eyes) with DON who underwent orbital decompression were included in the study. Two patients did not undergo preoperative OCT examination. Three additional patients were excluded owing to high intraocular pressure and high refractive error. Eight among 55 healthy controls were excluded because of high refractive error $(6$ patients), retinal lesion (1 patient), and glaucoma on ocular examination (1 patient). Two patients $(18 \%)$ with DON were male and 26 control subjects $(55 \%)$ were male $(p=$ 0.043 ). The mean age was $54 \pm 12$ years (mean $\pm \mathrm{SD}$, range, 33-70 years) at the time of surgery in patients with DON and $48 \pm 14$ years in healthy controls $(p=0.239$; Table 1$)$. All patients received intravenous methylprednisolone pulse therapy preoperatively, and their optic neuropathy was refractory to methylprednisolone therapy. No patient underwent radiation therapy before surgery. Two (18\%) patients underwent unilateral orbital decompression, and 9 (82\%) patients underwent bilateral decompression. No postoperative bleeding or infection of the orbital contents occurred. The mean time interval between the onset of DON and orbital decompression was $4 \pm 4$ months (range, 1 week-16 months). Three eyes in 2 patients underwent decompression surgery 6 months or later from the onset of DON. The other patients received decompression surgery within 6 months from disease onset.

The mean follow-up duration was $23 \pm 17$ months (range, 3-52 months). The mean LogMAR visual acuity improved from $0.5 \pm 0.5$ to $0.1 \pm 0.1$ at 1 month after the decompression surgery $(p<0.001)$. No patient experienced a decrease in visual acuity after surgery. In the measurements taken 6 months after intervention, mean improvement in LogMAR visual acuity was maintained as $0.1 \pm 0.2(p=$ $0.001)$. The mean ocular protrusion measured by a Hertel exophthalmometer was $15 \pm 3 \mathrm{~mm}$ at 1 month and $14 \pm 1$ $\mathrm{mm}$ at 6 months postoperatively, which decreased from 18 $\pm 4 \mathrm{~mm}$ preoperatively $(p<0.001)$. The mean color test score was $12 \pm 5$ at 1 month and $14 \pm 1$ at 6 months postoperatively, which increased from $5 \pm 6$ preoperatively $(p<$ 0.001 ). The mean deviation on the Humphrey field analyzer was $-1.9 \pm 4.1 \mathrm{~dB}$ at 1 month and $-1.1 \pm 1.8 \mathrm{~dB}$ at 6 months postoperatively, which improved from $-8.1 \pm 4.4$ $\mathrm{dB}$ preoperatively $(p<0.001)$. Three patients required postoperative radiotherapy for further stabilization of disease activity. Two patients underwent radiotherapy 6 months after the surgery, and one patient underwent radiotherapy 7 months after the surgery.

Table 2 presents peripapillary RNFL thicknesses in patients with DON before and after decompression surgery and in control subjects. There was no significant difference in mean preoperative peripapillary retinal nerve fiber layer thickness between eyes with dysthyroid optic neuropathy and controls. When peripapillary RNFL thicknesses was compared preoperatively vs. 1 month postoperatively, significant differences were observed in superior and inferior peripapillary RNFL thickness $(p=$ 0.043 and $p=0.022$, respectively). When peripapillary RNFL thickness was compared between preoperatively vs. 6 months postoperatively, significant differences were observed in global average, superior, temporal, and inferior peripapillary RNFL thickness $(p=0.015, p=$ $0.028, p=0.009$, and $p=0.006$, respectively). Figure 1 shows representative preoperatively and postoperative OCT results. 
Table 2 Descriptive statistics (mean $\pm \mathrm{SD}$ ) and statistical comparisons of peripapillary retinal nerve fiber layer thicknesses in eyes with healthy controls and dysthyroid optic neuropathy pre and postoperatively

Sector of peripapillary RNFL Controls $(n=94$ eyes)* DON $(n=11,20$ eyes)

\begin{tabular}{|c|c|c|c|c|c|c|}
\hline & Mean \pm SD & $\begin{array}{l}\text { Pre-op* Mean } \\
\pm \mathrm{SD}\end{array}$ & $\begin{array}{l}\text { Post-op } 1 \mathrm{M} \\
\text { Mean } \pm \text { SD }\end{array}$ & $\begin{array}{l}\text { Pre vs. postop. } \\
p \text {-value }{ }^{\dagger}\end{array}$ & $\begin{array}{l}\text { Post-op } 6 \text { M Mean } \pm \\
\text { SD }\end{array}$ & $\begin{array}{l}\text { Pre vs. postop. } \\
p \text {-value }\end{array}$ \\
\hline Global average & $97 \pm 8 \mu \mathrm{m}$ & $97 \pm 10 \mu \mathrm{m}$ & $95 \pm 8 \mu \mathrm{m}$ & 0.070 & $90 \pm 8 \mu \mathrm{m}$ & 0.010 \\
\hline Temporal & $73 \pm 12 \mu \mathrm{m}$ & $76 \pm 10 \mu \mathrm{m}$ & $77 \pm 11 \mu \mathrm{m}$ & 0.899 & $67 \pm 13 \mu \mathrm{m}$ & 0.003 \\
\hline Nasal & $69 \pm 12 \mu \mathrm{m}$ & $69 \pm 9 \mu \mathrm{m}$ & $68 \pm 9 \mu \mathrm{m}$ & 0.401 & $66 \pm 7 \mu \mathrm{m}$ & 0.104 \\
\hline Superior & $118 \pm 14 \mu \mathrm{m}$ & $120 \pm 24 \mu \mathrm{m}$ & $116 \pm 18 \mu \mathrm{m}$ & 0.043 & $111 \pm 14 \mu \mathrm{m}$ & 0.041 \\
\hline Inferior & $124 \pm 15 \mu \mathrm{m}$ & $125 \pm 18 \mu \mathrm{m}$ & $120 \pm 17 \mu \mathrm{m}$ & 0.022 & $115 \pm 18 \mu \mathrm{m}$ & 0.003 \\
\hline
\end{tabular}

DON dysthyroid optic neuropathy, Post-op postoperative, Pre-op preoperative, RNFL retinal nerve fiber layer, $1 M 1$ month, $6 M 6$ months

*There was no significant difference in preoperative peripapillary retinal nerve fiber layer thicknesses between eyes with dysthyroid optic neuropathy and controls.

${ }^{\dagger}$ Generalized estimating equation

In univariate analysis, preoperative inferior peripapillary RNFL thickness marginally significantly affected postoperative visual acuity at the last visit (Table 3). Patients with greater inferior peripapillary RNFL thickness tended to have marginally better postoperative visual acuity at the last visit $(p=0.062$, odds ratio $(\mathrm{OR})=0.924)$. The correlation regarding gender could not be evaluated owing to the skewed gender distribution of the study subjects.

Variables with a $p$-value of $<0.1$ in the univariate analysis were included in the multivariate analysis. In the multivariate analysis, patients with greater preoperative inferior peripapillary RNFL thickness tended to have better postoperative visual acuity at the last visit $(p=0.024, \mathrm{OR}=0.926)$.

\section{Discussion}

Any kind of compressive lesions within the orbit, especially when the lesion compresses the proximal optic nerve, can cause optic disc swelling in the acute stage. DON can also accompany varying degrees of optic disc swelling, up to $33 \%[12,13]$. In a previous OCT study on DON, the mean peripapillary RNFL thicknesses in all sectors was 3-7 $\mu \mathrm{m}$ higher in patients with acute DON compared to controls [16]. However, the difference did not reach statistical significance. In that study, temporal peripapillary RNFL thickness was less in patients with chronic DON compared to controls and patients with acute DON. In the present study, the mean preoperative peripapillary RNFL thickness was almost identical between patients with DON and controls in each sector. The difference in mean peripapillary RNFL thickness did not exceed $3 \mu \mathrm{m}$ between patients with acute DON and controls. However, when peripapillary RNFL thickness was compared between pre- and 1 month postoperatively, superior and inferior peripapillary RNFL thickness decreased significantly at 1 month postoperatively compared to preoperative values $(p=0.043$ and $p=0.022$, respectively). When peripapillary RNFL thickness was compared between pre- and 6 months postoperatively, the global average, superior, temporal, and inferior peripapillary RNFL thickness decreased at 6 months postoperatively compared to preoperative values $(p=0.015, p=0.028, p$ $=0.009$, and $p=0.006$, respectively). On the contrary, visual functions, including visual acuity and visual field test results, improved at 1 and 6 months after intervention compared to preoperative measurements.

In DON, retinal ganglion cell (RGC) loss may occur as a result of retrograde axonal degeneration [20]. Axons in the damaged optic nerve can break up into fragments and start to disintegrate and disappear within several weeks in animal and human models [21, 22]. Optic atrophy and a resultant decrease in peripapillary RNFL thickness can occur when this phenomenon reaches the axons within the eye [23, 24]. This process can take 3-6 months depending on the nature and severity of the injury [25-27]. In this study, the mean time interval between the onset of DON and orbital decompression was 4 months. We postulate that the reason that the mean preoperative RNFL thickness in patients with DON was similar to that in controls was the mixed effect of optic nerve swelling and optic atrophy in our study subjects. The nerve fiber layer around the optic nerve might be affected by both retrograde degeneration and disc swelling. After decompressive surgery, axonal swelling may decrease and postoperative peripapillary RNFL thickness might reflect the amount of true optic nerve atrophy. A previous study that investigated the effect of optic nerve decompression using optic nerve sheath fenestration in patients with intracranial hypertension on peripapillary RNFL thickeness also revealed that the average RNFL thickness decreased to $103 \mu \mathrm{m}$ from $114 \mu \mathrm{m}$ postoperatively [28]. Also, delayed ganglion cell atrophy could lead to a progressive decrease in peripapaillary RNFL thickness 
Fig. 1 Representative preoperative (top) and 1 (middle) and 6 months postoperative (bottom) peripapillary optical coherence tomography taken from a 70year old patient with bilateral dysthyroid optic neuropathy (DON) who underwent bilateral orbital decompression
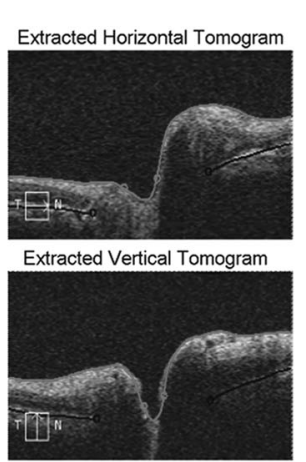

RNFL Circular Tomogram
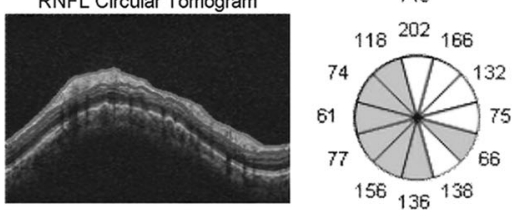

RNFL Thickness
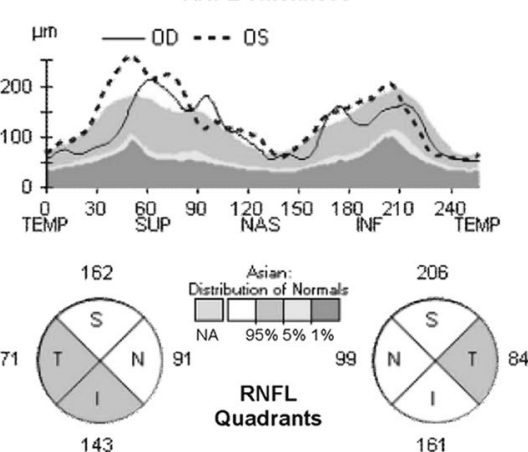

161

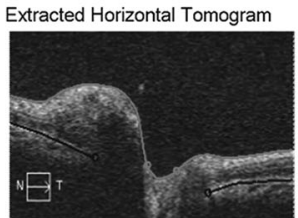

Extracted Vertical Tomogram

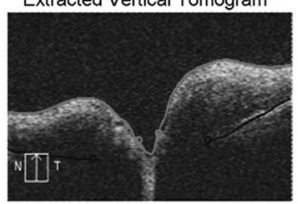

RNFL Circular Tomogram

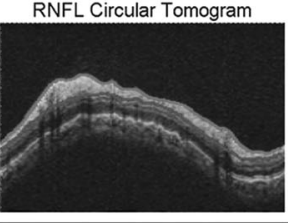

RNFL Thickness

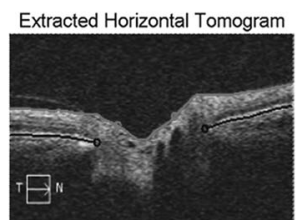

Extracted Vertical Tomogram

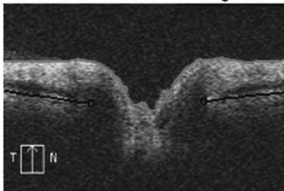

RNFL Circular Tomogram
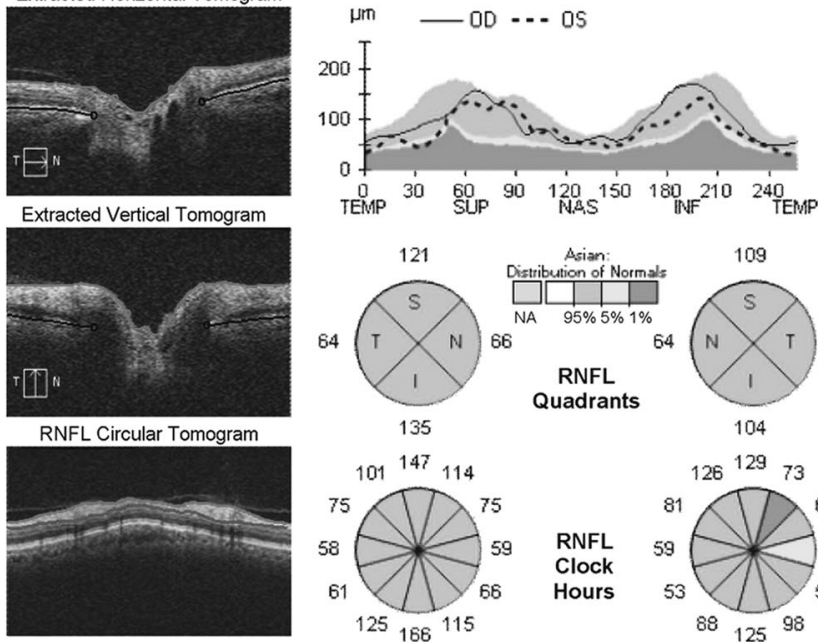

135

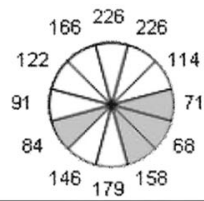

Extracted Horizontal Tomogram

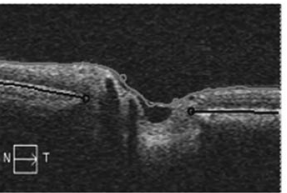

Extracted Vertical Tomogram
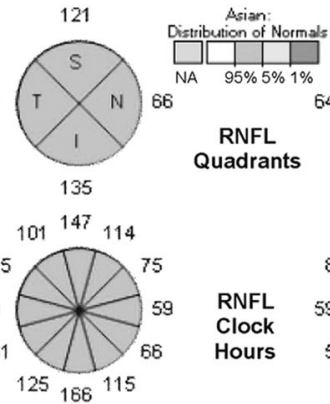

109
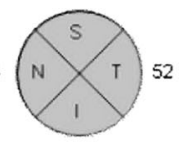

104

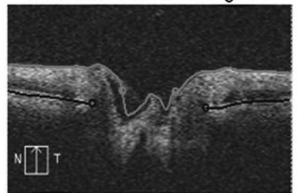

RNFL Circular Tomogram
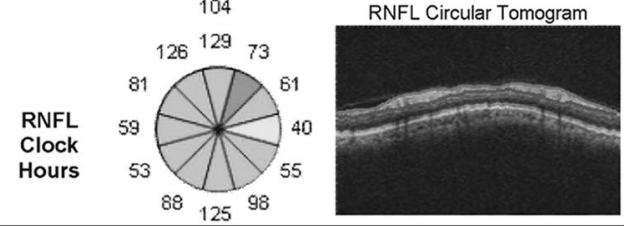

RNFL Thickness

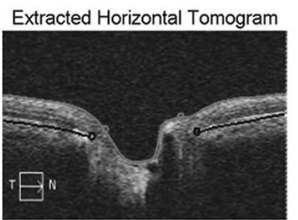

Extracted Vertical Tomogram
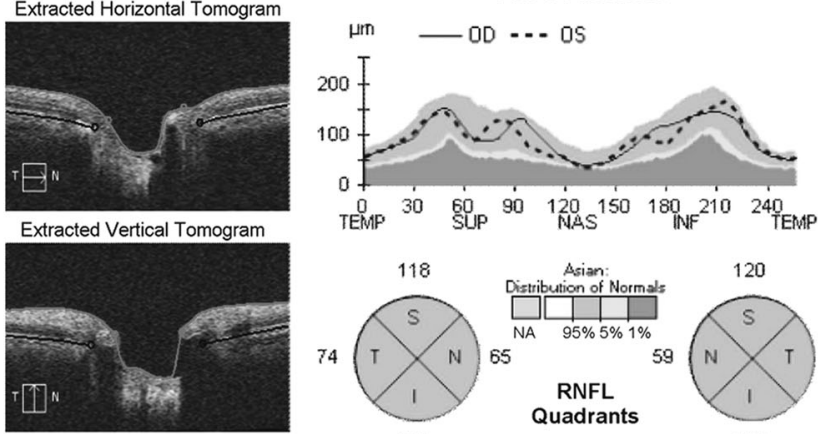

Extracted Horizontal Tomogram

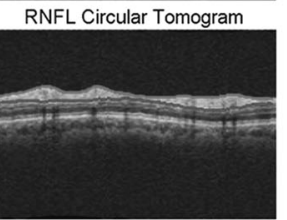

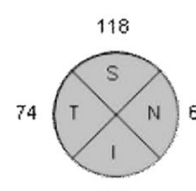

126

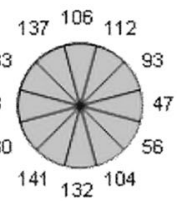

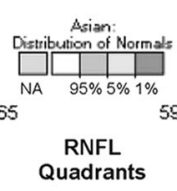

Quadrants

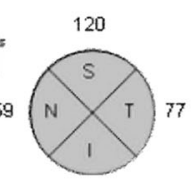

123

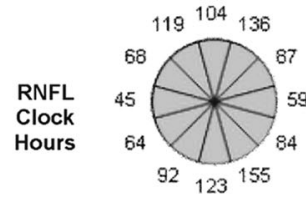

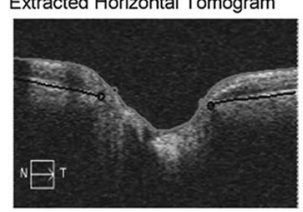

Extracted Vertical Tomogram

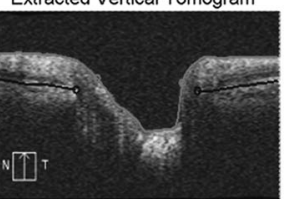

RNFL Circular Tomogram

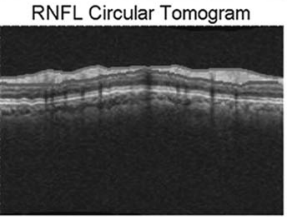

postoperatively. Prolonged retinal ganglion cell atrophy after decompression surgery has also been reported in cases of compressive optic neuropathy due to tumors [29].
In the multivariable analysis, patients with greater preoperative inferior peripapillary RNFL thickness tended to have better postoperative visual acuity at the last visit. This 
Table 3 Univariate analysis of baseline characteristics and optical coherence tomography measurements with better visual acuity at the last visit in dysthyroid optic neuropathy

\begin{tabular}{lllll}
\hline Baseline characteristics & $\begin{array}{l}\text { Odds } \\
\text { ratio }\end{array}$ & $\begin{array}{l}\text { 95\% CI of } \\
\text { odds ratio }\end{array}$ & $p$-value* \\
\hline Age & 1.094 & 0.983 & 1.218 & 0.101 \\
Gender $^{\dagger}$ & - & - & - & - \\
$\begin{array}{l}\text { Initial best-corrected visual } \\
\text { acuity }\end{array}$ & 5.399 & 0.762 & 38.265 & 0.092 \\
Color vision test (Ishihara) & 1.012 & 0.853 & 1.201 & 0.891 \\
score & & & & \\
Hertel exophthalmometry & 0.993 & 0.732 & 1.347 & 0.962 \\
Mean deviation on Humphrey & 1.052 & 0.853 & 1.297 & 0.638 \\
Field Analyzer & & & & \\
Peripapillary RNFL thicknesses & & & & \\
Global average & 0.929 & 0.843 & 1.023 & 0.136 \\
Temporal & 1.085 & 0.983 & 1.197 & 0.105 \\
Nasal & 0.961 & 0.872 & 1.059 & 0.417 \\
Superior & 0.989 & 0.963 & 1.014 & 0.381 \\
Inferior & 0.924 & 0.850 & 1.004 & 0.062 \\
\hline
\end{tabular}

$C I$ confidence interval, $R N F L$ retinal nerve fiber layer

*Univariate analysis using the generalized estimating equation

${ }^{\dagger}$ The correlation regarding gender could not be evaluated due to skewed distribution in this study subjects.

result is similar to the result from a previous study on patients with DON treated with various methods [16]. Greater inferior RNFL thickness may be a reflection of disc swelling caused by optic nerve compression, which may suggest acute DON. It may also suggest that there is no significant thinning of RNFL in the chronic stage of DON, which means no significant permanent damage to the optic nerve.

This study had several limitations. First, it was retrospective in design. Second, the sample size was small. We cannot evaluate the specific effect of combined treatment modalities to the OCT measurements. Third, we used Snellen visual acuity chart and transformed the visual acuity to a logarithmic scale for statistical analysis. Using specific logMAR chart is ideal for the analysis relating to visual acuities. Fourth, we conducted visual field test using the 30-2 SITA-standard protocol. The test for peripheral field was not conducted. Fifth, we used data from a single center and a population of Asian ethnicity. Some of the results may not be valid in other ethnic groups. Further, large-scale, prospective studies are needed to confirm the postoperative changes in OCT parameters and correlations with other ocular parameters.

In conclusion, the results of this study showed a significant decrease in peripapillary RNFL thickness postoperatively after orbital decompression surgery in patients with DON. The decrease in peripapillary RNFL thickness lasted until 6 months after decompression surgery. We also found a significant association between preoperative inferior peripapillary RNFL thickness and postoperative visual outcomes. Greater preoperative inferior peripapillary RNFL thickness was associated with better visual outcomes. We suggest that RNFL thickness can be used as a prognostic factor for DON before decompression surgery. Further studies with larger sample sizes using a prospective design should more clearly reveal the changes in peripapillary RNFL thickness after decompression surgery in patients with DON and the clinical significance thereof.

\section{Summary}

\section{What was known before}

The authors previously found that temporal peripapillary retinal nerve fiber layer (RNFL) thickness was less in patients with chronic DON compared to controls and patients with acute DON using optical coherence tomography (OCT). There was a significant association between RNFL thickness and visual acuity after treatment for dysthyroid orbitopathy. However, in this study, heterogeneous treatment methods were applied and many patients received treatment for DON before OCT examination. As a result, the authors could not evaluate the effect of each treatment modality on the OCT measurements.

\section{What this study adds}

The results of this study showed a significant decrease in peripapillary RNFL thickness postoperatively after orbital decompression surgery in patients with DON. The decrease in peripapillary RNFL thickness lasted until 6 months after decompression surgery. We also found a significant association between preoperative inferior peripapillary RNFL thickness and postoperative visual outcomes. Greater preoperative inferior peripapillary RNFL thickness was associated with better visual outcomes. We suggest that RNFL thickness can be used as a prognostic factor for DON before decompression surgery.

Author contributions The following authors were involved; study design (Y.D.K.); conduction of the study (K.A.P., Y.D.K.); data collection and management (K.A.P., Y.D.K.); data analysis and interpretation (K.A.P.); drafting the manuscript (K.A.P.); and the review and final approval of the manuscript (K.I.W., Y.D.K.). This study followed the tenets of the Declaration of Helsinki. This study was approved by the Institutional Review Board of Samsung Medical Center.

\section{Compliance with ethical standards}

Conflict of interest The authors declare that they have no conflict of interest. 


\section{References}

1. Carter KD, Frueh BR, Hessburg TP, Musch DC. Long-term efficacy of orbital decompression for compressive optic neuropathy of Graves' eye disease. Ophthalmology. 1991;98: 1435-42.

2. McNab AA. Orbital decompression for thyroid orbitopathy. Aust N Z J Ophthalmol. 1997;25:55-61.

3. Garrity JA, Fatourechi V, Bergstralh EJ, Bartley GB, Beatty CW, DeSanto LW, et al. Results of transantral orbital decompression in 428 patients with severe Graves' ophthalmopathy. Am J Ophthalmol. 1993;116:533-47.

4. Feldon SE, Muramatsu S, Weiner JM. Clinical classification of Graves' ophthalmopathy. Identification of risk factors for optic neuropathy. Arch Ophthalmol. 1984;102:1469-72.

5. Dosso A, Safran AB, Sunaric G, Burger A. Anterior ischemic optic neuropathy in Graves' disease. J Neuroophthalmol. 1994; $14: 170-4$.

6. Koorneef L, Schmidt ED. The orbit: structure, autoantigens, and pathology. In: Wall J, How J, editors. Graves' Ophthalmopathy. Oxford: Blackwell Scientific Publications; 1990.

7. Lane CM, Boschi A. Management of very severe Graves' orbitopathy (dysthyroid optic neuropathy and corneal breakdown). In: Wiersinga WM, Kahaly GJ, editors. Graves Orbitopathy: A Multidisciplinary Approach. Basel: Karger; 2007.

8. Ben Simon GJ, Syed HM, Douglas R, Schwartz R, Goldberg RA, McCann JD. Clinical manifestations and treatment outcome of optic neuropathy in thyroid-related orbitopathy. Ophthalmic Surg Lasers Imaging. 2006;37:284-90.

9. Liao SL, Chang TC, Lin LL. Transcaruncular orbital decompression: an alternate procedure for Graves ophthalmopathy with compressive optic neuropathy. Am J Ophthalmol. 2006;141: 810-8.

10. Perry JD, Kadakia A, Foster JA. Transcaruncular orbital decompression for dysthyroid optic neuropathy. Ophthalmol Plast Reconstr Surg. 2003;19:353-8.

11. Metson R, Dallow RL, Shore JW. Endoscopic orbital decompression. Laryngoscope. 1994;104:950-7.

12. Trobe JD, Glaser JS, Laflamme P. Dysthyroid optic neuropathy. Clinical profile and rationale for management. Arch Ophthalmol. 1978;96:1199-209.

13. Hutchison BM, Kyle PM. Long-term visual outcome following orbital decompression for dysthyroid eye disease. Eye (Lond). 1995;9(Pt 5):578-81.

14. McKeag D, Lane C, Lazarus JH, Baldeschi L, Boboridis K, Dickinson AJ, et al. Clinical features of dysthyroid optic neuropathy: a European Group on Graves' Orbitopathy (EUGOGO) survey. Br J Ophthalmol. 2007;91:455-8.
15. Wood-Allum CA, Shaw PJ Thyroid disease and the nervous system. In: Biller J, Ferro JM (eds). Neurologic Aspects of Systemic Disease Part II. Elsevier B.V., San Diego; 2014.

16. Park KA, Kim YD, In Woo K, Kee C, Han JC. Optical coherence tomography measurements in compressive optic neuropathy associated with dysthyroid orbitopathy. Graefes Arch Clin Exp Ophthalmol 2016;254:1617-24.

17. Bendschneider D, Tornow RP, Horn FK, Laemmer R, Roessler CW, Juenemann AG, et al. Retinal nerve fiber layer thickness in normals measured by spectral domain OCT. J Glaucoma. 2010;19:475-82.

18. Hwang YH, Lee SM, Kim YY, Lee JY, Yoo C. Astigmatism and optical coherence tomography measurements. Graefes Arch Clin Exp Ophthalmol. 2012;250:247-54.

19. Zeger SL, Liang KY. Longitudinal data analysis for discrete and continuous outcomes. Biometrics. 1986;42:121-30.

20. Duchen LW. General pathology of neurons and neuroglia. In: Adams JH, Duchen LW, editors. Greenfield's Neuropathology. 5th ed. New York: Oxford University Press; 1992. p. 1-68.

21. Sadun AA, Schaechter JD. Tracing axons in the human brain: a method utilizing light and TEM techniques. J Electron Microsc Tech. 1985;2:175-86.

22. Johnson BM, Sadun AA. Ultrastructural and paraphenylene studies of degeneration in the primate visual system: degenerative remnants persist for much longer than expected. J Electron Microsc Tech. 1988;8:179-83.

23. Anderson DR. Ascending and descending optic atrophy produced experimentally in squirrel monkeys. Am J Ophthalmol. 1973; 76:693-711.

24. Radius RL, Anderson DR. Retinal ganglion cell degeneration in experimental optic atrophy. Am J Ophthalmol. 1978;86:673-9.

25. Allcutt D, Berry M, Sievers J. A quantitative comparison of the reactions of retinal ganglion cells to optic nerve crush in neonatal and adult mice. Brain Res. 1984;318:219-30.

26. Barron KD, Dentinger MP, Krohel G, Easton SK, Mankes R. Qualitative and quantitative ultrastructural observations on retinal ganglion cell layer of rat after intraorbital optic nerve crush. J Neurocytol. 1986;15:345-62.

27. Grafstein B, Ingoglia NA. Intracranial transection of the optic nerve in adult mice: preliminary observations. Exp Neurol. 1982;76:318-30.

28. Starks V, Gilliland G, Vrcek I, Gilliland C. Effect of optic nerve sheath fenestration for idiopathic intracranial hypertension on retinal nerve fiber layer thickness. Orbit. 2016;35:87-90.

29. Moon CH, Hwang SC, Ohn YH, Park TK. The time course of visual field recovery and changes of retinal ganglion cells after optic chiasmal decompression. Invest Ophthalmol Vis Sci. 2011;52:7966-73. 